PROCEEDINGS OF THE

AMERICAN MATHEMATICAL SOCIETY

Volume 128, Number 11, Pages 3317-3327

S 0002-9939(00)05405-8

Article electronically published on May 11, 2000

\title{
ON THE EXISTENCE OF DOUBLING MEASURES WITH CERTAIN REGULARITY PROPERTIES
}

\author{
PER BYLUND AND JAUME GUDAYOL \\ (Communicated by Dale Alspach)
}

\begin{abstract}
Given a compact pseudo-metric space, we associate to it upper and lower dimensions, depending only on the pseudo-metric. Then we construct a doubling measure for which the measure of a dilated ball is closely related to these dimensions.
\end{abstract}

\section{INTRODUCTION}

Let $(X, \rho)$ be a compact metric space. Suppose that $(X, \rho)$ is homogeneous. This means that there exists a doubling measure $\mu$ supported by $X$; i.e. there is a constant $c$ such that, for $x \in X$ and $R>0$, one has $0<\mu(B(x, R))<\infty$ and

$$
\mu(B(x, 2 R)) \leq c \mu(B(x, R)) .
$$

Dynkin proved in Dyn that for certain subsets $E$ of the unit sphere $\mathbb{T} \subset \mathbb{C}$ there exists a doubling measure on $E$, and he conjectured that any compact $E \subset \mathbb{R}^{n}$ is homogeneous. This conjecture was proved in $\mathrm{V}-\mathrm{K}$ by using a dimension first defined in Lar called the uniform metric dimension, in this paper denoted by $\Upsilon(E)$. More precisely, Volberg and Konyagin proved that $(X, \rho)$ is homogeneous if and only if there is some $s<\infty$ such that any ball $B(x, k R)$ contains at most $C k^{s}$ points separated from each other by a distance of at least $R$. The uniform metric dimension $\Upsilon(X)=\Upsilon(X, \rho)$ is then defined as the infimum of such $s$. Furthermore, given $s<\infty$ in the condition above Volberg and Konyagin proved that for any $s^{\prime}>s$ there exists a measure $\mu$ such that, for $0<R \leq k R$,

$$
\mu(B(x, k R)) \leq C k^{s^{\prime}} \mu(B(x, R)) .
$$

Clearly, any measure satisfying (2) is a doubling measure, and conversely, iterating (11) one gets (2) with $s^{\prime}=\log _{2} c$. In particular, Volberg and Konyagin proved Dynkin's conjecture by showing that on any compact $E \subset \mathbb{R}^{n}$ there exists a measure $\mu$ satisfying (2) with $s=n$ (in the maximum metric).

In this paper we generalize their result by showing the existence of a measure $\mu$ not only satisfying (2), but also the following analogous lower bound condition. Suppose there is a $t \geq 0$ such that any ball $B(x, k R)$ contains at least $C k^{t}$ points

Received by the editors May 14, 1998 and, in revised form, January 4, 1999.

2000 Mathematics Subject Classification. Primary 28C15; Secondary 54E45, 54F45.

The second author is partially supported by MEC grant PB95-0956-C02-01 and CIRIT grant GRQ94-2014. 
separated from each other by a distance of at least $R$. Then for any $t^{\prime}<t$ there exists a measure $\mu$ such that, for $0<R \leq k R$,

$$
C k^{t^{\prime}} \mu(B(x, R)) \leq \mu(B(x, k R)) .
$$

In $[\mathrm{J}-\mathrm{W}]$ Jonsson and Wallin studied function spaces on subsets of $\mathbb{R}^{n}$ supporting measures fulfilling both (2) and (3) in the special case $s^{\prime}=t^{\prime}$. Such sets are also called $s$-sets or Ahlfors-regular sets.

The general case $t^{\prime} \leq s^{\prime}$ was considered in Jon.

The authors of this paper, independently of each other, also studied the general case $t^{\prime} \leq s^{\prime}$ in $\left.\overline{\mathrm{Byl}}\right]$ and [Gud]. Each of these works contains the main result of this paper, in Byl formulated for Euclidean spaces and in Gud] for metric spaces.

In this paper the result is stated in terms of pseudo-metric spaces.

\section{Definitions And STATEMEnts of Results}

Throughout we denote by $X=(X, d)$ a compact pseudo-metric space, where $d: X \times X \mapsto[0,+\infty)$ is a pseudo-metric on $X$, i.e.

1. $d(x, y)=0 \Longleftrightarrow x=y$,

2. $d(x, y)=d(y, x), \quad \forall x, y \in X$,

3. there is a constant $C_{d}$ such that $d(x, z) \leq C_{d}(d(x, y)+d(y, z)), \forall x, y, z \in X$. On $X$ we consider the topology generated by the open pseudo-balls, and without loss of generality we assume that $\operatorname{diam}(X)<1$.

Given any ball $B(x, k R), x \in X$ and $0<R \leq k R$, denote by $N(x, R, k)$ the maximum number of points in $B(x, k R)$ separated by a distance greater than or equal to $R$ from each other.

Definition 1. Define $X \in \Upsilon_{s}$ if there exists $C=C(s)$ such that, for $0<R \leq k R$,

$$
N(x, R, k) \leq C k^{s} .
$$

The upper dimension $\Upsilon(X)$ is then defined as

$$
\Upsilon(X)=\inf \left\{s \mid X \in \Upsilon_{s}\right\} .
$$

$\Upsilon(X)$ was introduced in [Lar] called the uniform metric dimension.

Definition 2. A positive Borel measure $\mu \in U_{s}$ if there exists $C=C(s)$ such that, for $x \in X$ and $0<R \leq k R$,

$$
\mu(B(x, k R)) \leq C k^{s} \mu(B(x, R)) .
$$

The dimension $U(X)$ is then defined as

$$
U(X)=\inf \left\{s \mid U_{s} \neq \emptyset\right\} .
$$

Note that by taking $k=1 / R$ in $\left(U_{s}\right)$ one gets the weaker condition

$$
\mu(B(x, R)) \geq C R^{s}, \quad x \in X, \quad 0<R .
$$

Also note that if $\mu \in U_{s}$, for some $s$, then $\operatorname{supp}(\mu)=X$. As mentioned in the introduction, $\mu$ is doubling precisely when $\mu \in U_{s}$ for some $s<\infty$. We will write $\mathcal{U}=\bigcup_{s} U_{s}$ for the set of all doubling measures on $X$.

Volberg and Konyagin proved $([\mathrm{V}-\mathrm{K}])$ that $\Upsilon(X) \leq U(X)$, and furthermore:

Theorem 1 (Volberg-Konyagin). Let $X$ be a compact metric space. If $X \in \Upsilon_{s}$, then for any $s^{\prime}>s$ there exists a measure $\mu \in U_{s^{\prime}}$. Consequently, $\Upsilon(X)=U(X)$. 
Our main result is Theorem 2 extending Theorem 1 to the analogue lower dimension. Note that Theorem 2 is stated for pseudo-metric spaces.

We start by defining the concept of the lower dimension.

\section{The lower dimension.}

Definition 3. Define $X \in \Lambda_{t}$ if there exists $C=C(t)$ such that, for $x \in X$ and $0<R \leq k R$,

$$
N(x, R, k) \geq C k^{t} .
$$

The lower dimension $\Lambda(X)$ is then defined as

$$
\Lambda(X)=\sup \left\{t \mid X \in \Lambda_{t}\right\} .
$$

$\Lambda(X)$ was introduced in $\left([\underline{\mathrm{Lar}})\right.$ called the minimal dimension. Note that $X \in \Lambda_{0}$ is trivial.

Definition 4. A positive Borel measure $\mu \in L_{t}$ if there exists $C=C(t)$ such that, for $x \in X$ and $0<R \leq k R$,

$$
\mu(B(x, k R)) \geq C k^{t} \mu(B(x, R)) .
$$

As before, by taking $k=1 / R$ in $\left(L_{t}\right)$ one gets the weaker condition

$$
\mu(B(x, R)) \leq C R^{t}, \quad x \in X, \quad 0<R .
$$

Now, observe that defining the lower dimension as

$$
L(X)=\sup \left\{t \mid L_{t} \neq \emptyset\right\}
$$

will not work since $\mu \in L_{t}$ does not imply $\operatorname{supp}(\mu)=X$, so this will say nothing about $X \backslash \operatorname{supp}(\mu)$. The appropriate definition is as follows.

Definition 5. Define the lower dimension $L(X)$ as

$$
L(X)=\sup \left\{t \mid L_{t} \cap \mathcal{U} \neq \emptyset\right\} .
$$

Note that $L_{0}$ poses no restriction on $\mu \in \mathcal{U}$.

The main theorem. We now state the main result of this paper. Note that in the special case $t=0$ one can take $t^{\prime}=t=0$.

Theorem 2. Let $X \in \Upsilon_{s} \cap \Lambda_{t}, 0 \leq t \leq s<+\infty$, be a compact pseudo-metric space. Then for any $s^{\prime}>s$ and $t^{\prime}<\bar{t}$ there is a probability measure $\mu \in U_{s^{\prime}} \cap L_{t^{\prime}}$.

From Theorem 2 and Propositions 4 and 5 below we then get

Corollary 3. If $\Upsilon(X)<+\infty$, then $\Upsilon(X)=U(X)$ and $\Lambda(X)=L(X)$.

\section{Proof of the theorem}

To prove Theorem 2 we construct a sequence of measures with certain properties and the desired measure $\mu$ will be a limit point of this sequence.

We start by proving the trivial inequalities $\Upsilon(X) \leq U(X)$ and $\Lambda(X) \geq L(X)$. 


\subsection{The trivial inequalities.}

Proposition 4. If $\mu \in U_{s}$, then $X \in \Upsilon_{s}$, i.e. $\Upsilon(X) \leq U(X)$.

Proof. Let $\mu \in U_{s}$, fix any $x \in X$ and let $x_{1}, \ldots, x_{N}$ be points in $B(x, k R)$ with $d\left(x_{i}, x_{j}\right) \geq R$ for $i \neq j$. Since $\mu \in U_{s}$ and $B\left(x, 2 C_{d} k R\right) \subset B\left(x_{i}, 4 C_{d}^{2} k R\right)$,

$$
\mu\left(B\left(x, 2 C_{d} k R\right)\right) \leq \mu\left(B\left(x_{i}, 4 C_{d}^{2} k R\right)\right) \leq C 8^{s} C_{d}^{3 s} k^{s} \mu\left(B\left(x_{i}, \frac{R}{2 C_{d}}\right)\right) .
$$

Also, the balls $B\left(x_{i}, R /\left(2 C_{d}\right)\right)$ are disjoint and lie in $B\left(x, 2 C_{d} k R\right)$, so

$$
\mu\left(B\left(x, 2 C_{d} k R\right)\right) \geq \sum_{i=1}^{N} \mu\left(B\left(x_{i}, \frac{R}{2 C_{d}}\right)\right) \geq N \frac{\mu\left(B\left(x, 2 C_{d} k R\right)\right)}{C 8^{s} C_{d}^{3 s} k^{s}} .
$$

Thus $N \leq C k^{s}$, i.e. $\Upsilon(X) \leq U(X)$.

Proposition 5. If $\mu \in L_{t} \cap \mathcal{U}$, then $X \in \Lambda_{t}$, i.e. $\Lambda(X) \leq L(X)$.

Proof. Let $\left\{x_{1}, \ldots, x_{N}\right\}$ be a maximal set of points in $B(x, k R)$ separated by a distance greater than or equal to $R$. Fix any $\mu \in L_{t} \cap \mathcal{U}$. Then, since $\mu$ is doubling and $B\left(x_{i}, k R\right) \subset B\left(x, 2 C_{d} k R\right)$ for all $i$,

$$
C \mu(B(x, k R)) \geq \mu\left(B\left(x, 2 C_{d} k R\right)\right) \geq \mu\left(B\left(x_{i}, k R\right)\right) \geq C k^{t} \mu\left(B\left(x_{i}, R\right)\right) .
$$

Also, $B(x, k R) \subset \bigcup_{i=1}^{N} B\left(x_{i}, R\right)$, since $\left\{x_{1}, \ldots, x_{N}\right\}$ is maximal, i.e.

$$
\mu(B(x, k R)) \leq \sum_{i=1}^{N} \mu\left(B\left(x_{i}, R\right)\right) \leq \frac{N}{C k^{t}} \mu(B(x, k R)) .
$$

Thus, $N \geq C k^{t}$, i.e. $X \in \Lambda_{t}$.

3.2. The main lemma. Assume that $X \in \Lambda_{t} \cap \Upsilon_{s}$. Let $C_{d}$ be the constant associated to the pseudo-metric $d, C_{t}$ the constant appearing in $\Lambda_{t}$ and $C_{s}$ the one in $\Upsilon_{s}$. Given $t^{\prime}<t$ and $s^{\prime}>s$, choose $A \geq 16 C_{d}^{4}$ large enough such that $A^{s^{\prime}-s}>C_{s}$ and $A^{t-t^{\prime}}>4^{t} C_{d}^{2 t} C_{t}^{-1}$. For each non-negative integer $j$, let $S_{j}$ be a maximal set of points in $X$ separated by a distance greater than or equal to $A^{-j}$.

Define mappings $\mathcal{E}=\mathcal{E}_{m}: S_{m+1} \rightarrow S_{m}$ for $m \geq 0$ as follows. For $g \in S_{m+1}$ choose one of the points $e \in S_{m}$ for which $d(g, e)=d\left(g, S_{m}\right)$, and denote it by $e=\mathcal{E}(g)$. Then for $e \in S_{m}$ let

$$
S_{e, m+1}=\left\{g \in S_{m+1}, e=\mathcal{E}(g)\right\} .
$$

It is easy to see that $\left\{S_{e, m+1} \mid e \in S_{m}\right\}$ form a partition of $S_{m+1}$.

The desired measure $\mu$ will be a limit of measures $\mu_{m}$ supported by $S_{m}$. Lemma 7 below will allow us to perform the inductive step that constructs $\mu_{m+1}$ from $\mu_{m}$. First though we need the following preparatory lemma.

Lemma 6. Let $e \in S_{m}$. Then

$$
A^{t^{\prime}} \leq \#\left(S_{e, m+1}\right) \leq A^{s^{\prime}}
$$

where \# denotes the cardinality of a set.

Proof. Fix any $e \in S_{m}$. Clearly $S_{e, m+1} \subset B\left(e, A^{-m}\right)$ since $S_{m}$ is maximal. Therefore, and since $X \in \Upsilon_{s}$ and $A^{s^{\prime}-s}>C_{s}$,

$$
\#\left(S_{e, m+1}\right) \leq \#\left(S_{m+1} \cap B\left(e, A^{-m}\right)\right) \leq N\left(e, A^{-m-1}, A\right) \leq C_{s} A^{s} \leq A^{s^{\prime}},
$$

which proves the right inequality of the lemma. 
For the left inequality, we first note that there exists $g \in S_{m+1}$ for which $d(g, e)<$ $A^{-m-1}$, and as $A>2 C_{d}$ it is clear that $e=\mathcal{E}(g)$ for such $g$.

Also, for $e^{\prime} \neq e^{\prime \prime}$ we have $B\left(e^{\prime}, A^{-m} / 2 C_{d}\right) \cap B\left(e^{\prime \prime}, A^{-m} / 2 C_{d}\right)=\emptyset$. Thus,

$$
S_{m+1} \cap B\left(e, A^{-m} /\left(2 C_{d}\right)\right) \subset S_{e, m+1} .
$$

Next, for $\left\{g_{i}\right\}_{i=1}^{n}=S_{m+1} \cap B\left(e, A^{-m} / 2 C_{d}\right)$ we have

$$
n \geq N\left(e, A^{-m-1}, A / 2 C_{d}^{2}-1\right) .
$$

To check it, suppose the contrary, that is, suppose that

$$
n<N\left(e, A^{-m-1}, A / 2 C_{d}^{2}-1\right)=n_{1} .
$$

Then there would exist points $x_{1}, \ldots, x_{n_{1}}$ in $B\left(e,\left(A / 2 C_{d}^{2}-1\right) A^{-m-1}\right)$ separated from each other by a distance greater than or equal to $A^{-m-1}$.

But, for $g \in S_{m+1} \backslash\left(S_{m+1} \cap B\left(e, A^{-m} / 2 C_{d}\right)\right)$ we have

$$
d\left(g, x_{i}\right) \geq \frac{1}{C_{d}} d(g, e)-d\left(e, x_{i}\right) \geq \frac{A}{2 C_{d}^{2}} A^{-m-1}-\left(\frac{A}{2 C_{d}^{2}}-1\right) A^{-m-1}=A^{-m-1},
$$

which means that the set

$$
S_{m+1}^{\prime}=\left(\left\{x_{i}\right\}_{i=1}^{n_{1}} \cup S_{m+1}\right) \backslash\left(S_{m+1} \cap B\left(e, \frac{A}{2 C_{d}} A^{-m-1}\right)\right)
$$

fulfills $\#\left(S_{m+1}^{\prime}\right)>\#\left(S_{m+1}\right)$, a contradiction to the maximality of $S_{m+1}$.

Thus, from $(*),(\dagger)$, the choice of $A$ and the fact that $X \in \Lambda_{t}$, we conclude

$$
\begin{aligned}
\#\left(S_{e, m+1}\right) & \geq \#\left(S_{m+1} \cap B\left(e, A^{-m} / 2 C_{d}\right)\right) \geq N\left(e, A^{-m-1}, A / 2 C_{d}^{2}-1\right) \\
& \geq C_{t}\left(A / 2 C_{d}^{2}-1\right)^{t} \geq C_{t} A^{t}\left(4 C_{d}^{2}\right)^{-t} \geq A^{t^{\prime}} .
\end{aligned}
$$

Lemma 7. Let $f_{0}$ be a measure on $S_{m}$ such that for any $e, e^{\prime} \in S_{m}$ we have

$$
f_{0}\left(e^{\prime}\right) \leq C_{1} f_{0}(e)
$$

whenever $d\left(e, e^{\prime}\right) \leq C_{2} A^{-m}$, with $C_{1}=A^{s^{\prime}-t^{\prime}}$, and $C_{2}=8 C_{d}^{3}$. Then there is a measure $f_{1}$ on $S_{m+1}$ with the following properties:

(a) $f_{1}\left(g^{\prime}\right) \leq C_{1} f_{1}(g)$ for any $g, g^{\prime} \in S_{m+1}$ with $d\left(g, g^{\prime}\right) \leq C_{2} A^{-m-1}$.

(b) If $g \in S_{e, m+1}$, then $A^{-s^{\prime}} f_{0}(e) \leq f_{1}(g) \leq A^{-t^{\prime}} f_{0}(e)$.

(c) $f_{0}(X)=f_{1}(X)$.

(d) The construction of the measure $f_{1}$ from the measure $f_{0}$ can be regarded as a transfer of mass from the points in $S_{m}$ to those of $S_{m+1}$, with no mass transferred over a distance greater than $2 C_{d} A^{-m}$. This means that if $g \in$ $S_{m+1}$ receives mass from $e \in S_{m}$, then $d(g, e) \leq 2 C_{d} A^{-m}$.

Proof of the lemma. Let $f_{00}$ be the measure obtained by homogeneously distributing the mass of each $e \in S_{m}$ on the points in $S_{e, m+1}$. By doing so, we obtain a measure satisfying (b) (because of Lemma 6), (c) and (d). If $f_{00}$ satisfies (a), then let $f_{1}=f_{00}$ and we are done.

Assume that $f_{00}$ does not satisfy (a). Let $\left\{g_{i}^{\prime}, g_{i}^{\prime \prime}\right\}_{i=1}^{T}$ be all the pairs of points in $S_{m+1}$ with $d\left(g_{i}^{\prime}, g_{i}^{\prime \prime}\right) \leq C_{2} A^{-m-1}$. We will construct a finite sequence of measures $\left\{f_{0 j}, j=1, \ldots, T\right\}$, such that $f_{0 j}$ will satisfy (a) for all the pairs $\left\{\left(g_{i}^{\prime}, g_{i}^{\prime \prime}\right)\right\}_{i=1}^{j}$, and as we will see $f_{1}=f_{0 T}$ is the desired measure. 
The construction of $f_{0 j+1}$ from $f_{0 j}$ is as follows:

If $C_{1}^{-1} f_{0 j}\left(g_{j+1}^{\prime \prime}\right) \leq f_{0 j}\left(g_{j+1}^{\prime}\right) \leq C_{1} f_{0 j}\left(g_{j+1}^{\prime \prime}\right)$, then let $f_{0 j+1}=f_{0 j}$. Otherwise, only one of these inequalities can fail, and without loss of generality we may assume that $f_{0 j}\left(g_{j+1}^{\prime}\right)>C_{1} f_{0 j}\left(g_{j+1}^{\prime \prime}\right)$. Then we move mass from $g_{j+1}^{\prime}$ to $g_{j+1}^{\prime \prime}$ by defining $f_{0 j+1}$ as

$$
\begin{aligned}
f_{0 j+1}\left(g_{j+1}^{\prime}\right) & =f_{0 j}\left(g_{j+1}^{\prime}\right)-\frac{f_{0 j}\left(g_{j+1}^{\prime}\right)-C_{1} f_{0 j}\left(g_{j+1}^{\prime \prime}\right)}{C_{1}+1} ; \\
f_{0 j+1}\left(g_{j+1}^{\prime \prime}\right) & =f_{0 j}\left(g_{j+1}^{\prime \prime}\right)+\frac{f_{0 j}\left(g_{j+1}^{\prime}\right)-C_{1} f_{0 j}\left(g_{j+1}^{\prime \prime}\right)}{C_{1}+1} ; \\
f_{0 j+1}(g) & =f_{0 j}(g) \quad \text { if } g \notin\left\{g_{j+1}^{\prime}, g_{j+1}^{\prime \prime}\right\} .
\end{aligned}
$$

With this definition $f_{0 j+1}\left(g_{j+1}^{\prime}\right)=C_{1} f_{0 j+1}\left(g_{j+1}^{\prime \prime}\right)$, which means that (a) is true for $f_{0 j+1}$ with respect to $\left(g_{j+1}^{\prime}, g_{j+1}^{\prime \prime}\right)$. In particular, note that (a) is true for $f_{01}$ with respect to $\left(g_{1}^{\prime}, g_{1}^{\prime \prime}\right)$.

We are now going to check condition (b) for $f_{0 j+1}$. To do so, suppose that (b) holds for $f_{0 j}$, i.e. suppose that

$$
A^{-s^{\prime}} f_{0}(e) \leq f_{1}(g) \leq A^{-t^{\prime}} f_{0}(e), \quad g \in S_{e, m+1} .
$$

If $f_{0 j+1}=f_{0 j}$ or $g \notin\left\{g_{j+1}^{\prime}, g_{j+1}^{\prime \prime}\right\}$, then there is nothing to check. Otherwise, as before we can assume that $f_{0 j}\left(g_{j+1}^{\prime}\right)>C_{1} f_{0 j}\left(g_{j+1}^{\prime \prime}\right)$. Let $e^{\prime}=\mathcal{E}\left(g_{j+1}^{\prime}\right)$ and $e^{\prime \prime}=\mathcal{E}\left(g_{j+1}^{\prime \prime}\right)$. It is clearly enough to prove that $f_{0 j+1}\left(g_{j+1}^{\prime}\right) \geq A^{-s^{\prime}} f_{0}\left(e^{\prime}\right)$ and $f_{0 j+1}\left(g_{j+1}^{\prime \prime}\right) \leq A^{-t^{\prime}} f_{0}\left(e^{\prime \prime}\right)$ (because $f_{0 j+1}\left(g_{j+1}^{\prime}\right)<f_{0 j}\left(g_{j+1}^{\prime}\right) \leq A^{-t^{\prime}} f_{0}\left(e^{\prime}\right)$ and $\left.f_{0 j+1}\left(g_{j+1}^{\prime \prime}\right)>f_{0 j}\left(g_{j+1}^{\prime \prime}\right) \geq A^{-s^{\prime}} f_{0}\left(e^{\prime \prime}\right)\right)$. Now

$$
\begin{aligned}
d\left(e^{\prime}, e^{\prime \prime}\right) & \leq C_{d} d\left(e^{\prime}, g_{j+1}^{\prime}\right)+C_{d}^{2} d\left(g_{j+1}^{\prime}, g_{j+1}^{\prime \prime}\right)+C_{d}^{2} d\left(g_{j+1}^{\prime \prime}, e^{\prime \prime}\right) \\
& \leq C_{d} A^{-m}+C_{2} C_{d}^{2} A^{-m-1}+C_{d}^{2} A^{-m} \leq C_{2} A^{-m}
\end{aligned}
$$

so $f_{0}\left(e^{\prime}\right) \leq C_{1} f_{0}\left(e^{\prime \prime}\right)$. Therefore

$$
\begin{aligned}
f_{0 j+1}\left(g_{j+1}^{\prime \prime}\right) & =C_{1}^{-1} f_{0 j+1}\left(g_{j+1}^{\prime}\right) \leq C_{1}^{-1} f_{0 j}\left(g_{j+1}^{\prime}\right) \\
& \leq C_{1}^{-1} A^{-t^{\prime}} f_{0}\left(e^{\prime}\right) \leq A^{-t^{\prime}} f_{0}\left(e^{\prime \prime}\right) .
\end{aligned}
$$

Analogously, $f_{0}\left(e^{\prime \prime}\right) \geq C_{1}^{-1} f_{0}\left(e^{\prime}\right)$. Thus,

$$
\begin{aligned}
f_{0 j+1}\left(g_{j+1}^{\prime}\right) & =C_{1} f_{0 j+1}\left(g_{j+1}^{\prime \prime}\right) \geq C_{1} f_{0 j}\left(g_{j+1}^{\prime \prime}\right) \\
& \geq C_{1} A^{-s^{\prime}} f_{0}\left(e^{\prime \prime}\right) \geq A^{-s^{\prime}} f_{0}\left(e^{\prime}\right) .
\end{aligned}
$$

Consequently, since (b) holds for $f_{00}$ it is clear that it holds for $f_{1}=f_{0 T}$ as well.

We are now going to check that when a pair satisfies (a) with respect to $f_{0 j}$, it also does with respect to $f_{0 j+1}$. To this end, pick any pair $\left(g_{1}, g_{2}\right), d\left(g_{1}, g_{2}\right) \leq$ $C_{2} A^{-m-1}$, for which

$$
C_{1}^{-1} f_{0 j}\left(g_{1}\right) \leq f_{0 j}\left(g_{2}\right) \leq C_{1} f_{0 j}\left(g_{1}\right) .
$$

If $\left(g_{1}, g_{2}\right)$ and $\left(g_{j+1}^{\prime}, g_{j+1}^{\prime \prime}\right)$ have no point in common or if $f_{0 j+1}=f_{0 j}$, then we are done. Otherwise, $f_{0 j+1} \neq f_{0 j}$ and $f_{0 j}\left(g_{j+1}^{\prime}\right)>C_{1} f_{0 j}\left(g_{j+1}^{\prime \prime}\right)$. Then the two pairs have only one point in common, say $g_{1}$. In this case $f_{0 j+1}\left(g_{2}\right)=f_{0 j}\left(g_{2}\right)$. 
We have two possible cases to consider, either $g_{1}=g_{j+1}^{\prime}$ or $g_{1}=g_{j+1}^{\prime \prime}$ :

1. If $g_{1}=g_{j+1}^{\prime \prime}$, then $f_{0 j+1}\left(g_{1}\right)>f_{0 j}\left(g_{1}\right)$. Thus, in this case it is enough to prove that $f_{0 j+1}\left(g_{1}\right) \leq C_{1} f_{0 j+1}\left(g_{2}\right)$. Let $e^{\prime}=\mathcal{E}\left(g_{j+1}^{\prime}\right)$ and $e_{2}=\mathcal{E}\left(g_{2}\right)$. Then

$$
\begin{gathered}
d\left(e^{\prime}, e_{2}\right) \leq C_{d} d\left(e^{\prime}, g_{j+1}^{\prime}\right)+C_{d}^{3} d\left(g_{j+1}^{\prime}, g_{1}\right)+C_{d}^{3} d\left(g_{1}, g_{2}\right)+C_{d}^{2} d\left(g_{2}, e_{2}\right) \\
\leq C_{d} A^{-m}+2 C_{d}^{3} C_{2} A^{-m-1}+C_{d}^{2} A^{-m} \leq C_{2} A^{-m},
\end{gathered}
$$

so $f_{0}\left(e^{\prime}\right) \leq C_{1} f_{0}\left(e_{2}\right)$. Also, since we already know that (b) is true, we have $f_{0}\left(e_{2}\right) \leq A^{s^{\prime}} f_{0 j+1}\left(g_{2}\right)$ and $f_{0 j+1}\left(g_{j+1}^{\prime}\right) \leq A^{-t^{\prime}} f_{0}\left(e^{\prime}\right)$. Thus,

$$
\begin{aligned}
f_{0 j+1}\left(g_{1}\right) & =f_{0 j+1}\left(g_{j+1}^{\prime \prime}\right)=C_{1}^{-1} f_{0 j+1}\left(g_{j+1}^{\prime}\right) \leq C_{1}^{-1} A^{-t^{\prime}} f_{0}\left(e^{\prime}\right) \\
& \leq A^{-t^{\prime}} f_{0}\left(e_{2}\right) \leq A^{s^{\prime}-t^{\prime}} f_{0 j}\left(g_{2}\right)=A^{s^{\prime}-t^{\prime}} f_{0 j+1}\left(g_{2}\right)=C_{1} f_{0 j+1}\left(g_{2}\right) .
\end{aligned}
$$

2. Otherwise, if $g_{1}=g_{j+1}^{\prime}$, then $f_{0 j+1}\left(g_{1}\right)<f_{0 j}\left(g_{1}\right)$. Thus, it is enough to check that $f_{0 j+1}\left(g_{1}\right) \geq C_{1}^{-1} f_{0 j+1}\left(g_{2}\right)$. But, for $e^{\prime \prime}=\mathcal{E}\left(g_{j+1}^{\prime \prime}\right)$, then as in (4), $d\left(e^{\prime \prime}, e_{2}\right) \leq C_{2} A^{-m}$. Also, $f_{0 j+1}\left(g_{1}\right)=C_{1} f_{0 j+1}\left(g_{j+1}^{\prime \prime}\right)$. Thus, from (b) we then get

$$
\begin{aligned}
f_{0 j+1}\left(g_{1}\right) & =C_{1} f_{0 j+1}\left(g_{j+1}^{\prime \prime}\right) \geq C_{1} A^{-s^{\prime}} f_{0}\left(e^{\prime \prime}\right) \geq A^{-s^{\prime}} f_{0}\left(e_{2}\right) \\
& \geq A^{t^{\prime}-s^{\prime}} f_{0 j+1}\left(g_{2}\right)=C_{1}^{-1} f_{0 j+1}\left(g_{2}\right) .
\end{aligned}
$$

This concludes the proof that (a) is true for $f_{1}$.

Clearly $f_{0 j+1}(X)=f_{0 j}(X)$, so (c) is also true for $f_{1}$.

It remains to check (d). When passing from $f_{0}$ to $f_{00}$ no mass is moved over a distance exceeding $A^{-m}$, because $S_{e, m+1} \subset B\left(e, A^{-m}\right)$, and when going from $f_{0 j}$ to $f_{0 j+1}$ no mass is moved over a distance exceeding $C_{2} A^{-m-1}$, and $C_{2} / A<1$. It therefore remains to prove that in the construction of $f_{1}$ from $f_{0}$ there are no pairs $\left(g_{1}, g_{2}\right)$ and $\left(g_{2}, g_{3}\right)$ in $S_{m+1}$ for which mass is first moved from $g_{1}$ to $g_{2}$ and then at a subsequent step from $g_{2}$ to $g_{3}$. To prove this, assume the opposite. Then

$$
f_{00}\left(g_{1}\right)>C_{1} f_{00}\left(g_{2}\right) \quad \text { and } \quad f_{00}\left(g_{2}\right)>C_{1} f_{00}\left(g_{3}\right) .
$$

But, if $e_{1}=\mathcal{E}\left(g_{1}\right)$ and $e_{3}=\mathcal{E}\left(g_{3}\right)$, then as in (4), $d\left(e_{1}, e_{3}\right) \leq C_{2} A^{-m}$, so by the hypothesis $C_{1}^{-1} f_{0}\left(e_{1}\right) \leq f_{0}\left(e_{3}\right) \leq C_{1} f_{0}\left(e_{1}\right)$. Also,

$$
A^{-s^{\prime}} f_{0}\left(e_{i}\right) \leq f_{00}\left(g_{i}\right) \leq A^{-t^{\prime}} f_{0}\left(e_{i}\right),
$$

for $i=1$ and $i=3$. Adding these two inequalities, we would then get

$$
f_{0}\left(e_{1}\right) \geq A^{t^{\prime}} f_{00}\left(g_{1}\right)>C_{1} A^{t^{\prime}} f_{00}\left(g_{2}\right)>C_{1}^{2} A^{t^{\prime}} f_{00}\left(g_{3}\right) \geq C_{1}^{2} A^{t^{\prime}-s^{\prime}} f_{0}\left(e_{3}\right),
$$

contradicting $f_{0}\left(e_{1}\right) \leq C_{1} f_{0}\left(e_{3}\right)$, as $d\left(e_{1}, e_{3}\right) \leq C_{2} A^{-m}$ and $C_{1}=A^{s^{\prime}-t^{\prime}}$.

3.3. Proof of the theorem. We will now use Lemma 7 to construct a sequence of probability measures and prove that any limit point of this sequence belongs to $L_{t^{\prime}} \cap U_{s^{\prime}}$.

We start by defining a probability measure $\mu_{0}$ on $S_{0}$ (note that $S_{0}$ consists of one point only, by the assumption $\operatorname{diam}(X)<1$ ). Obviously $\mu_{0}$ satisfies the hypothesis of Lemma 7. By using Lemma 7 to construct $\mu_{j+1}=f_{1}$ on $S_{j+1}$ from $\mu_{j}=f_{0}$, $j \geq 0$, we then get a sequence $\left\{\mu_{j}\right\}_{j=0}^{\infty}$ of probability measures. This sequence belongs to the unit ball of the dual of the Banach space $\mathcal{C}(X)$, and thus has at least one weak limit point. Let $\mu$ be any limit point of this sequence. In the proof we will frequently use the following proposition, based on (d) of Lemma 7 . 
Proposition 8. Let $j \in \mathbb{N}, r \geq 0, x \in X$ and put $C_{3}=2 C_{d}^{2} /\left(1-C_{d} / A\right)$. Then

$$
\mu_{j}(B(x, r)) \leq \mu\left(B\left(x, r+C_{3} A^{-j}\right)\right)
$$

and

$$
\mu(B(x, r)) \leq \mu_{j}\left(B\left(x, r+C_{3} A^{-j}\right)\right) .
$$

Proof. According to (d) of Lemma 7 no mass is moved at a distance exceeding $2 C_{d} A^{-j}$ when constructing $\mu_{j+1}$ from $\mu_{j}$. Thus, when passing from $\mu_{j}$ to $\mu_{j+k}$, $k \geq 1$, no mass is moved at a distance exceeding

$$
2 C_{d}^{2} A^{-j} \sum_{n=0}^{k-1}\left(C_{d} / A\right)^{n}<\frac{2 C_{d}^{2}}{1-C_{d} / A} A^{-j}=C_{3} A^{-j},
$$

which means that there is no mass transfer from $B(x, r)$ into the complement of $B\left(x, r+C_{3} A^{-j}\right)$, and vice versa. Thus,

$$
\mu_{j}(B(x, R)) \leq \mu_{j+k}\left(B\left(x, r+C_{3} A^{-j}\right)\right)
$$

and

$$
\mu_{j+k}(B(x, r)) \leq \mu_{j}\left(B\left(x, r+C_{3} A^{-j}\right)\right) .
$$

Now, as $\mu$ is a weak limit point of $\left\{\mu_{j+k}\right\}$, the same is true for $\mu$ as well.

We will now prove that $\mu \in L_{t^{\prime}} \cap U_{s^{\prime}}$. To this end, fix $x \in X$ and some $R$ and $k$ for which $0<R \leq k R$. Then choose integers $m$ and $M$ such that

$$
k R \leq A^{-m}<A k R \quad \text { and } \quad \frac{R}{A} \leq A^{-M}<R .
$$

Denote by $e_{M+1}$ one of the points in $S_{M+1}$ closest to $x$ (there may be several) and for $j=0, \ldots, M-m$ define $e_{M-j}=\mathcal{E}\left(e_{M-j+1}\right) \in S_{M-j}$.

First claim.

$$
\mu_{m+2}\left(e_{m+2}\right) \leq \mu(B(x, k R)) \leq C_{s} 3^{s^{\prime}}\left(1+C_{3}\right)^{s} C_{1} \mu_{m}\left(e_{m}\right) .
$$

Proof. By the definition of $e_{M-j}$ and property 3 of the pseudo-metric $d$, we have

$$
d\left(x, e_{m+2}\right) \leq C_{d} A^{-m-2} \sum_{j=0}^{\infty}\left(C_{d} / A\right)^{j}=\frac{C_{d}}{1-C_{d} / A} A^{-m-2} .
$$

Let $y \in B\left(e_{m+2}, C_{3} A^{-m-2}\right)$. Then, by (5),

$$
d(y, x) \leq C_{d} C_{3} A^{-m-2}+\frac{C_{d}^{2}}{1-C_{d} / A} A^{-m-2} \leq A^{-m-1}<k R,
$$

i.e. $B\left(e_{m+2}, C_{3} A^{-m-2}\right) \subset B(x, k R)$. From Proposition 8 we then get

$$
\mu_{m+2}\left(e_{m+2}\right) \leq \mu\left(B\left(e_{m+2}, C_{3} A^{-m-2}\right)\right) \leq \mu(B(x, k R)),
$$

proving the left inequality in (6). To prove the right inequality, note that (55) and Proposition 8 imply

$$
\mu(B(x, k R)) \leq \mu_{m}\left(B\left(x, k R+C_{3} A^{-m}\right)\right) \leq \mu_{m}\left(B\left(x,\left(1+C_{3}\right) A^{-m}\right)\right) .
$$

But, $d\left(x, e_{m}\right) \leq \frac{C_{d}}{1-C_{d} / A} A^{-m}$. Thus, if $e \in S_{m} \cap B\left(x,\left(1+C_{3}\right) A^{-m}\right)$, then

$$
d\left(e, e_{m}\right) \leq C_{d}\left(1+C_{3}\right) A^{-m}+\frac{C_{d}^{2}}{1-C_{d} / A} A^{-m} \leq C_{2} A^{-m},
$$


so from Lemma 7 it follows that $\mu_{m}(e) \leq C_{1} \mu_{m}\left(e_{m}\right)$. Now,

$$
\text { \# }\left(S_{m} \cap B\left(x,\left(1+C_{3}\right) A^{-m}\right)\right) \leq C_{s}\left(1+C_{3}\right)^{s},
$$

so from Proposition 8 and the fact that $k R \leq A^{-m}$, we get

$$
\mu(B(x, k R)) \leq \mu_{m}\left(B\left(x,\left(1+C_{3}\right) A^{-m}\right)\right) \leq C_{s}\left(1+C_{3}\right)^{s} C_{1} \mu_{m}\left(e_{x, m}\right),
$$

which concludes the proof of the first claim.

\section{Second claim.}

$$
\mu_{M+1}\left(e_{M+1}\right) \leq \mu(B(x, R)) \leq C_{s}\left(1+C_{3}\right)^{s^{\prime}} A^{2 s^{\prime}} C_{1} \mu_{M+1}\left(e_{M+1}\right) .
$$

Proof. By the definition of $e_{M+1}$,

$$
d\left(e_{M+1}, x\right)=d\left(x, S_{M+1}\right) \leq A^{-M-1}<R / A .
$$

Thus, for $y \in B\left(e_{x, M+1}, C_{3} A^{-M-1}\right)$,

$$
d(y, x) \leq C_{d} C_{3} A^{-M-1}+C_{d} A^{-M-1} \leq A^{-M}<R .
$$

Again by Proposition 8 ,

$$
\mu_{M+1}\left(e_{M+1}\right) \leq \mu\left(B\left(e_{M+1}, C_{3} A^{-M-1}\right)\right) \leq \mu(B(x, R)),
$$

proving the left inequality in (77). To prove the right inequality, note that from Proposition 8 and the fact that $R \leq A^{-M+1}$, by the choice of $M$,

$$
\mu(B(x, R)) \leq \mu_{M-1}\left(B\left(x, R+C_{3} A^{-M+1}\right)\right) \leq \mu_{M-1}\left(B\left(x,\left(1+C_{3}\right) A^{-M+1}\right)\right) .
$$

Also, for $g \in B\left(x, R+C_{3} A^{-M+1}\right) \cap S_{M-1}$,

$$
\begin{aligned}
& d\left(g, e_{M-1}\right) \leq C_{d} d(g, x)+C_{d}^{3} d\left(x, e_{M+1}\right)+C_{d}^{3} d\left(e_{M+1}, e_{M}\right)+C_{d}^{2} d\left(e_{M}, e_{M-1}\right) \\
& \quad \leq C_{d}\left(1+C_{3}\right) A^{-M+1}+C_{d}^{3} A^{-M-1}+C_{d}^{3} A^{-M}+C_{d}^{2} A^{-M+1} \leq C_{2} A^{-M+1} .
\end{aligned}
$$

Thus, from (a) and (b) of Lemma 7 we get (recalling $e_{M-j}=\mathcal{E}\left(e_{M-j+1}\right)$ ),

$$
\mu_{M-1}(g) \leq C_{1} \mu_{M-1}\left(e_{M-1}\right) \leq C_{1} A^{2 s^{\prime}} \mu_{M+1}\left(e_{M+1}\right) .
$$

But,

$$
\text { \# }\left(B\left(x,\left(1+C_{3}\right) A^{-M+1}\right) \cap S_{M-1}\right) \leq C_{s}\left(1+C_{3}\right)^{s},
$$

so

$$
\mu(B(x, R)) \leq \mu_{M-1}\left(B\left(x,\left(1+C_{3}\right) A^{-M+1}\right)\right) \leq C_{s}\left(1+C_{3}\right)^{s} A^{2 s^{\prime}} C_{1} \mu_{M+1}\left(e_{M+1}\right),
$$

proving the second claim. To conclude the proof, note that

$\mu\left(e_{m}\right) \leq A^{s^{\prime}(M+1-m)} \mu_{M+1}\left(e_{M+1}\right) \quad$ and $\quad \mu_{m+2}\left(e_{m+2}\right) \geq A^{t^{\prime}(M-m-1)} \mu_{M+1}\left(e_{M+1}\right)$

by (b) in Lemma 7 Also note that $k<A^{M-m} \leq A^{2} k$, by the choice of $m$ and $M$.

Thus, from the two claims it follows that

$$
\mu(B(x, k R)) \leq C \mu_{m}\left(e_{m}\right) \leq C A^{s^{\prime}(M-m)} \mu_{M+1}\left(e_{M+1}\right) \leq C k^{s^{\prime}} \mu(B(x, R)),
$$

and similarly,

$$
\mu(B(x, k R)) \geq \mu_{m+2}\left(e_{m+2}\right) \geq C A^{t^{\prime}(M-m)} \mu_{M+1}\left(e_{M+1}\right) \geq C k^{t^{\prime}} \mu(B(x, R)),
$$

i.e. $\mu \in \Lambda_{t^{\prime}} \cap \Upsilon_{s^{\prime}}$. 
Note that the final constants $C$ depend only on the given constants $C_{d}, C_{s}, C_{t}$ and the choice of $A, s^{\prime}$ and $t^{\prime}$. Also note that the last inequality depends on the fact that $\Upsilon(X)<+\infty$.

\section{THE NON-COMPACT CASE}

In [L-S] Theorem 1 was generalized to a non-compact complete metric space $X$. It is easy to see that their proof holds for a pseudo-metric, too. We conclude this paper by showing that Theorem 2 combined with their proof gives the analogue generalization of Theorem 2 as well. Before that we just briefly sketch their proof, and refer to $[\mathrm{L}-\mathrm{S}]$ for details:

Let $s^{\prime}>s$ and cover $X \in \Upsilon_{s}$ with a countable collection of compact balls $X_{n}=B\left(x_{0}, n\right), n \in \mathbb{N}, x_{0} \in X$. Every $X_{n}$ carries a $\mu_{n} \in U_{s^{\prime}}$, by Theorem 1 .

By using the weak-* compactness of the unit ball of $\mathcal{C}\left(X_{n}\right)$ and a Cantor's diagonal process they show the existence of a subsequence $\left\{\mu_{j}^{*}\right\}$ of $\left\{\mu_{n}\right\}$ such that, for every continuous $f \geq 0$ with compact support on $X_{p}, \int_{X_{p}} f d \mu_{j}^{*}$ converges to $\int_{X} f d \mu$ for some $\mu \in U_{s^{\prime}}$.

Theorem 9. Let $X \in \Upsilon_{s} \cap \Lambda_{t}$ be any complete pseudo-metric space. Then there exists a $\mu \in U_{s^{\prime}} \cap L_{t^{\prime}}$ for every $t^{\prime}<t$ and $s^{\prime}>s$.

Proof. We use the notation above. It remains to prove $\mu \in L_{t^{\prime}}$. From Theorem 2 it is clear that $\mu_{j}^{*} \in U_{s^{\prime}} \cap L_{t^{\prime}}$, where the constant $C$ in $L_{t^{\prime}}$ is the same for all $j$. Let $x \in X, r>0, k>1$. Let $0<\varepsilon<(k-1) /(k+1)$ and pick continuous functions $0 \leq f, g \leq 1$ such that $f=1$ on $B(x,(1-\varepsilon) k r)$ and $g=1$ on $B(x, r)$, and such that $f$ and $g$ have compact support on $B(x, k r)$ and $B(x,(1+\varepsilon) r)$, respectively. Put $c^{-1}=C k^{t^{\prime}}((1-\varepsilon) /(1+\varepsilon))^{t^{\prime}}$, choose $p$ such that $B(x, k r) \subset X_{p}$ and choose $j$ large enough that $\left|\int_{X_{p}} h d \mu-\int_{X_{p}} h d \mu_{j}^{*}\right|<\varepsilon$ for $h=f, g$. Then

$$
\begin{array}{r}
\mu(B(x, r)) \leq \int_{X_{p}} g d \mu \leq \int_{X_{p}} g d \mu_{j}^{*}+\varepsilon \leq \mu_{j}^{*}(B(x,(1+\varepsilon) r))+\varepsilon \\
\leq c \mu_{j}^{*}(B(x,(1-\varepsilon) k r))+\varepsilon \leq c \int_{X_{p}} f d \mu_{j}^{*}+\varepsilon \\
\leq c \int_{X_{p}} f d \mu+c \varepsilon+\varepsilon \leq c \mu(B(x, k r))+c \varepsilon+\varepsilon
\end{array}
$$

Letting $\varepsilon \rightarrow 0$ gives $C k^{t^{\prime}} \mu\left(B(x, r) \leq \mu\left(B(x, k r)\right.\right.$, i.e. $\mu \in L_{t^{\prime}}$.

\section{REFERENCES}

[Byl] Per Bylund, Besov spaces and measures on arbitrary closed sets, Doctoral Thesis No 8, 1994, Department of Mathematics, University of Umeå, Sweden. MR 95k:46046

[Dyn] E. M. Dynkin, Free interpolation by functions with derivatives in $H^{1}$, J. Soviet Math. 27 (1984), 2475-2481.

[Gud] Jaume Gudayol, Boundary behaviour of functions in Hardy-Sobolev spaces, doctoral dissertation, Universitat de Barcelona, 1997.

[Jon] Alf Jonsson, Besov spaces on closed subsets of $\mathbb{R}^{n}$, Transactions of the AMS 41 (1994), no. 1, 355-370. MR 94c:46065

[J-W] Alf Jonsson and Hans Wallin, Function spaces on subsets of $\mathbb{R}^{n}$, Math. Reports Volume 2, Part 1, Harwood Academics Publ. GmbH, 1984. 
[Lar] D.G. Larman, A new theory of dimension, Proc. London Math. Soc. (3rd series) 17 (1967), 178-192. MR 34:3540

[L-S] J. Luukkainen and E. Saksman, Every complete doubling metric space carries a doubling measure, Proceedings of the AMS 126 (1998), 531-534. MR 99c:28009

[V-K] A.L. Vol'berg and S.V. Konyagin, On measures with the doubling condition, Math. USSR Izvestiya 30 (1988), 629-638. MR 88i:28006

Department of Mathematics, University of Ume̊̊, S-90187 Ume̊̊, Sweden

E-mail address: Per.Bylund@math.umu.se

Departament de Matèmatica Aplicada i Anàlisi, Universitat de Barcelona, Gran Via 585, 08007 BARCELONA, SPAin

E-mail address: gudayol@mat.ub.es 